\title{
SWI as a promising tool comparable to CT perfusion in evaluation of acute cerebral infarction
}

\author{
Manal F. Abu-samra', Mohammed F. Amin ${ }^{1 *} \mathbb{D}$, Ahmed M. Yassen ${ }^{1}$, Asmaa K. Fath El-Bab ${ }^{1}$ and \\ Mohammed Farouk Gabr²
}

\begin{abstract}
Background: The recent advances in magnetic resonance imaging techniques have improved the assessment of acute stroke. Susceptibility weighted imaging (SWI) has a crucial role in the management plan of cerebral ischemia. This study was aimed to assess the role of susceptibility-weighted imaging in assessment of area at risk (pneumbra) compared to CT perfusion in patients with acute ischemic infraction.

Results: We found the mean aspect score for SWI $4 \pm 1.4$ and mean aspect for DWI 7.6 \pm 1.2 ; in addition, mean aspect for CTP was $4.6 \pm 1.3$. Significant difference is noted between the SWI and DWI with significant $p$ value. But there is no significant difference between the SWI and CTP ASPECT scores.
\end{abstract}

Conclusion: SWI is a promising technique and comparable to $C T$ perfusion is evaluation of penumbra in the settings of acute infarction.

Keywords: MRI, SWI, MRA, Arterial occlusion, Stroke, Infarction

\section{Background}

Ischemic stroke is one of the most common of the worldwide diseases with high mortality and disability rates [1]. The key to treating ischemic stroke is saving the ischemic penumbra. For patients with large vascular occlusion after acute cerebral infarction, the shorter the onset time, the less necrotic tissue and the more healthy tissue that can be saved [2].

However, recent research suggests that the rate of irreversible death of ischemic neurons after stroke is highly variable. This is influenced by a number of factors, such as the location of blood vessels and collateral circulation, which affect the death of ischemic neurons [3]; therefore, timely assessment of the penumbra tissue is the key to choosing which patients are ideally suited to reperfusion

\footnotetext{
*Correspondence: Mohammed_amin37@yahoo.com

${ }^{1}$ Radiology Department, Faculty of Medicine, Minia University, Minya,

Egypt

Full list of author information is available at the end of the article
}

and to enable an accurate prognosis. Perfusion-diffusion mismatch is the first choice in identifying the ischemic penumbra from the infarct core region $[4,5]$.

Contemporary therapy for acute ischemic stroke is based on the concept of penumbra, which is an area with reduced blood flow but not to such a level that causes irreversible cell membrane failure. Although challenged by several limitations, mismatch between larger abnormal areas on CT perfusion and MR perfusion-weighted imaging (PWI) and smaller restricted areas on diffusionweighted imaging (DWI) is a widely accepted approach to detecting penumbra $[6,7]$.

Predicting stroke evolution determines patients with the greatest potential to benefit from thrombolytic therapy, but CT perfusion and PWI require administration of a contrast agent, which is contra-indicated in a variety of clinical conditions, such as renal insufficiency and previous reactions to contrast agents [7-9]. 
Susceptibility-weighted imaging (SWI) is a highresolution, three-dimensional, gradient-echo T2* MR technique with enhanced sensitivity for paramagnetic substances, such as blood products, iron, and calcifications $[10,11]$. SWI has also been used in evaluating trauma, vascular malformations, tumours, as well as cerebrovascular and neurodegenerative diseases [12]. In acute ischemic stroke, severe reduction in cerebral perfusion pressure causes increased deoxy-haemoglobin to oxy-haemoglobin ratio by increasing oxygen extraction fraction (OEF) [13]. SWI can show asymmetric prominent hypo-intense vessels potentially from different concentrations of deoxy-haemoglobin between ischemic and normal brain areas. This potential metabolic information on SWI may help to delineate penumbra without contrast agent administration $[7,14,15]$.

It was hypothesized that SWI provides comparable perfusion information as $C T$ perfusion does and the SWI-DWI mismatch represents an alternative tool for evaluating ischaemic penumbra in patients with ischaemic stroke [7].

\section{Aim of the work}

The aim of this study is to assess the role of susceptibility-weighted imaging in assessment of area at risk (penumbra) compared to CT perfusion in patients with acute ischemic infraction.

\section{Methods}

\section{Study design and population}

This is retrospective study was done between December 2018 and June 2020; it included 25 patients (18 males and 7 females, age range was from 30 to 70 years) with a mean age of 60 year), with clinically diagnosed acute stroke. All patient were referred from the neurology department to our department. The examination was done after the approval of ethical committee of our institution.

\section{Inclusion and exclusion criteria \\ Inclusion criteria}

- All patients included in this study were diagnosed clinically having acute stroke.

- Age older than 18 years.

- Stroke symptoms lasting $>1 \mathrm{~h}$.

- National Institutes of Health Stroke Scale (NIHSS) score is more than 4 .

\section{Exclusion criteria}

General contraindications to MRI as the presence of any paramagnetic substances such as pacemakers, metallic clips or claustrophobic patients were excluded from the study. Patients with an NIHSS score less than or equal to 4 were excluded.

\section{Methods}

CT scanning

CT was performed with a CT HiSpeed 16 slice scanner (GE Medical Systems, Milwaukee, WI) in the hospital emergency department using the nonhelical scanning technique: $120 \mathrm{kV}, 300 \mathrm{~mA}, 1 \mathrm{~s}$ scanning time, and 5-mm section thickness.

\section{MRI technique}

Magnetic resonance imaging studies were performed with a 1.5-T MR system (Achieva, Philips). All patients underwent examination by our MRI protocol tailored specifically for stroke patients, which included the following fast imaging sequences: axial fast field echo (FFE) T1, axial turbo spin-echo (TSE) T2, FLAIR, and DWI, in addition to the SWI sequence and MRA. Various parameters for the basic MR sequences are listed in Table 1.

CT Perfusion (CTP) images were performed on GE MDCT 16 detectors; the iodinated contrast media passing through the brain were tracked using the timeresolved scans; and serial images (20-40) are taken over the same section of anatomy. The patients must be able to tolerate the examination avoiding motion. Injection rates

Table 1 Technique for MRI sequences, DWI and SWI

\begin{tabular}{|c|c|c|c|c|c|c|}
\hline & TR & TE & Flip angle & FOV & Slice thickness & Pixel spacing \\
\hline T1WI & 7.3 & $3.4 \mathrm{~ms}$ & 30 & 220 & $5 \mathrm{~mm}$ & $1-2 \mathrm{~mm}$ \\
\hline FLAIR & $600 \mathrm{~ms}$ & $11.0 \mathrm{~s}$ & 90 & 220 & $5 \mathrm{~mm}$ & $1-2 \mathrm{~mm}$ \\
\hline T2 & $4000 \mathrm{~ms}$ & $98 \mathrm{~ms}$ & 90 & 220 & $5 \mathrm{~mm}$ & $1-2 \mathrm{~mm}$ \\
\hline SWI & $52 \mathrm{~s}$ & $12 \mathrm{~ms}$ & 15 & $230 \times 190 \times 120$ & $0.5 \mathrm{~mm}$ & $1-2 \mathrm{~mm}$ \\
\hline MRA & 22 & 3.59 & 18 & $240 \times 200$ & 0.5 & $1-2$ \\
\hline DWI & 4045 & 127 & 90 & 231 & $5 \mathrm{~mm}$ & $1-2 \mathrm{~mm}$ \\
\hline
\end{tabular}


of 3 to $3.5 \mathrm{~mL} / \mathrm{s}$ were used. The scan is repeated every second to every $2-3 \mathrm{~s}$ for about $40-90 \mathrm{~s}$. Image thickness is relatively wide about $5-10 \mathrm{~mm}$ to avoid noise. The data are used to make colour maps representing the changes of brain circulation known as blood flow, blood volume, mean transient time, and time to perfusion peak.

The dose used is $80 \mathrm{kV}$ using low dose for each scan to avoid high cumulative dose [8].

\section{Image interpretation}

All images are read by two well qualified radiologist (15 years experience in reading CTP and MRI post-training) in consensus.

The SWI is actually used in our study to evaluate the area at risk (penumbra) mismatch with DWI comparing with CT perfusion images.

The acute infarction was identified in initial DW images as an area of bright signal intensity which appeared dark on the corresponding ADC map. Also affected area shows increased level of deoxy-haemoglobin in draining vessels so they will appear more hypo-intense in SWI and based on the SWI is able to detect the affected area as asymmetrical prominent cortical veins compared with the other normal site (APCV).

To do this, we compare between the SWI and brain perfusion. The Alberta Stroke Program Early CT Score (ASPECTS) was used to assess the extent of the ischemic area described above in the DW, SW, and CTP images.

According to the ASPECTS scoring system, the MCA territory is divided into ten zones: caudate nucleus, lentiform nucleus, internal capsule, insula, M1, M2, and M3 (anterior, middle, and posterior third of the lower MCA territory, respectively), and M4, M5, and M6 (anterior, middle, and posterior third of the higher MCA territory, respectively). To calculate the ASPECTS score in the initial DW, SW, and CTP images for each patient, 1 point was deducted from 10 for each area of restricted diffusion, APCVs, colour change, and hyperintensity in the DW, SW, and CTP images, respectively [8].

The ischemic area in the cerebral perfusion will result in changes in MTT, TTP, and CBV and CBF. Thus, we evaluate the area at risk by DWI/perfusion mismatch and then will compare DWI/CTP with DWI/SWI using ASPECT for each one.

\section{The results of comparison have four different scenarios}

(a) The affected brain tissues should still be viable and are likely to recover after treatment:

1. Acute infarction in DWI, while both SWI and T2-weighted imaging (T2WI) appear normal. Although MTT is delayed, CBV and CBF still remain normal, and in some cases it is even increased, known as compensatory over-perfusion after acute ischemic stroke.

2. Acute infarctions shown in DWI may show APCV in whole hemisphere on SWI, and the area covered matched the delays seen in MTT and TTP. The CBF slightly decreased, but CBV remained within the normal range this known as compensatory normal perfusion after acute ischemic stroke.

(b) The affected brain tissue is nonfunctioning and necrotic

3. The acute infarction seen on DWI shows no APCV, and the local MTT and TTP changes combined with affected $\mathrm{CBV}$ and $\mathrm{CBF}$. This affected brain tissue likely was compromised already and may well represent nonfunctioning tissue.

4. The presence of a large lobar hyper-intensity on DWI, but no veins were seen with SWI in the corresponding area along with a consistent region of delayed MTT and TTP that matched a decreased CBV and CBF; this tissue likely exposed to prolonged hypoxia and the tissue became necrotic $[9,16]$.

\section{Statistical analysis}

Statistical analysis was performed using the SPSS software for Windows v. 20 (SPSS Inc., Chicago, IL). Tests of significance (repeated measures ANOVA, Cochran's Q tests, Kappa statistics, and ROC Curve analysis) was used. $p$ values less than $0.05(5 \%)$ were considered to be statistically significant. Mean, standard deviation $( \pm S D)$, and range were used for parametric numerical data, while median and inter-quartile range (IQR) for non-parametric numerical data. While median and inter-quartile range (IQR) and also frequency and percentage for nonparametric numerical data.

\section{Results}

In our study, CTP images were done for all patients included in the study (25 patient) in addition to SWI \& DWI using ASPECT (Table 2) to assess extension of infarction in CTP, SWI, and DWI without bias, and we found the mean aspect score for SWI $4 \pm 1.4$ and mean aspect for DWI 7.6 \pm 1.2 ; in addition, mean aspect for CTP was $4.6 \pm 1.3$. From these results, we found there is significance difference between the SWI and DWI with significant $p$ value. On the other hand, there is 
Table 2 Aspect score for patients

\begin{tabular}{llll}
\hline Case & SWI aspect & DWI aspect & PWI aspect \\
\hline 1 & 5 & 9 & 5 \\
2 & 3 & 6 & 4 \\
3 & 4 & 7 & 5 \\
4 & 4 & 6 & 5 \\
5 & 3 & 7 & 3 \\
6 & 5 & 8 & 6 \\
7 & 6 & 9 & 6 \\
8 & 4 & 7 & 4 \\
9 & 7 & 9 & 7 \\
10 & 5 & 8 & 5 \\
11 & 4 & 7 & 3 \\
12 & 3 & 6 & 4 \\
13 & 3 & 7 & 3 \\
14 & 5 & 9 & 6 \\
15 & 4 & 9 & 3 \\
16 & 3 & 5 & 4 \\
\hline
\end{tabular}

Table 3 SWI changes versus DWI changes

\begin{tabular}{lcc}
\hline & Mean \pm SD & $p$ \\
\hline SWI & $4 \pm 1.4$ & $<0.0001$ \\
DWI & $7.6 \pm 1.2$ & \\
\hline
\end{tabular}

Table 4 SWI changes versus PWI changes

\begin{tabular}{lll}
\hline & Mean \pm SD & $\boldsymbol{p}$ \\
\hline SWI & $4.2 \pm 1.2$ & 0.5 \\
PWI & $4.6 \pm 1.3$ & \\
\hline
\end{tabular}

no significance difference between the SWI and CTP ASPECT scores (Fig. 1, 2).

All patients with APVS changes had CTP changes so 16 out of 19 patients with CTP had APVS representing 84\%, and only 3 has negative APVS. On the other hand, no one with negative CTP had APVS; this proves that there is
Table 5 Comparison between PWI/DWI mismatch and SWI and diffusion mismatch

\begin{tabular}{llll}
\hline & $\mathbf{N}=\mathbf{2 5}$ & & $\boldsymbol{p}$ \\
\cline { 2 - 3 } & PWI/DWI mismatch & SWI/DWI mismatch & \\
\hline Positive & $19(76 \%)$ & $16(64 \%)$ & 0.5 \\
Negative & $6(24 \%)$ & $9(36 \%)$ & \\
Total & 25 & 25 & \\
\hline
\end{tabular}

Table 6 Relation between PWI changes and APVS in SWI

\begin{tabular}{lllll}
\hline PWI & APVS & No APVS & Total & $\boldsymbol{p}$ \\
\hline Positive & $16(84 \%)$ & $3(16 \%)$ & 19 & 0.001 \\
Negative & $0(0 \%)$ & $6(100 \%)$ & 6 & \\
Total & 16 & 9 & 25 & \\
\hline
\end{tabular}

good correlation between the CTP and APVS with significant $p$ value (Fig. 3).

As regarding ASPECT score in different pulse sequences including SWI, DWI, and perfusion-weighted images, there was no discrepancy between SWI and CTP which show the same number in many patients. On the other hand, the DWI shows discrepancy in its numbers compared to SWI and CTP (Table 3).

There is significance difference between SWI ASPECT and DWI ASPECT scores with significant $p$ value (Table 4).

No significance difference between SWI and CTP ASPECT score with insignificant $\mathrm{P}$ value was noted (Table 5).

In total, 19 patients have perfusion-diffusion mismatch representing about $76 \%$ compared to 16 patients who have susceptibility-diffusion mismatch representing $64 \%$ with no significant difference $p$ value (Table 6).

Only 16 out of 19 patients who have perfusion changes show APVS in SWI and the remaining patients 6 have negative APVS proving there is correlation between the CTP changes and APVS with significant $p$ value (Table 6).

\footnotetext{
(See figure on next page.)

Fig. 1 58-year-old male presented to stroke unit with left-sided weakness of 3-h duration, a DWI reveals normal signal intensity of both cerebral hemispheres. No definite areas of diffusion restriction. ASPECT score 10, CTP $\mathbf{b}$ revealed significant delay in mean transient time along the left frontotemporoparietal region (arrows) as well as the insula; however, deep nuclei are not affected compared to right side. The affected segments M1-6 and insula representing ASPECT score 3, c SWI reveals asymmetrical vessel sign with prominent dark dilated veins on the left cerebral hemisphere compared to right hemisphere along M1 to M6 segments; however, the deep nuclei and insula are not affected representing ASPECT score 4. The ASPECT score of SWI is very close to perfusion aspect score; on the other hand, the DWI reveals no abnormality. ASPECT score (3, 4 \& 10), respectively. So there is DW/P mismatch denoting there is an area of risk to infarction (penumbra). Also there is DW/SWI mismatch similar to perfusion mismatch. This supports the role of SWI in detection of area at risk. D. SWI of the same patient revealed asymmetrical vessel sign in the form prominent cortical veins (arrow head) on the left cerebral hemisphere along (M1-M6 segments) representing the area at risk with aspect score 4
} 


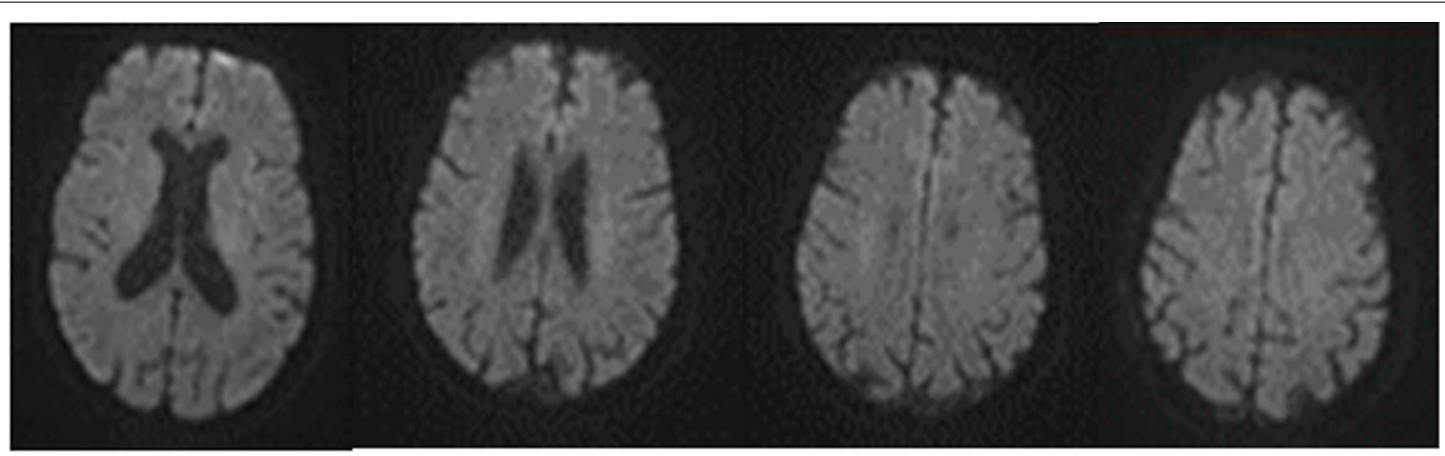

A

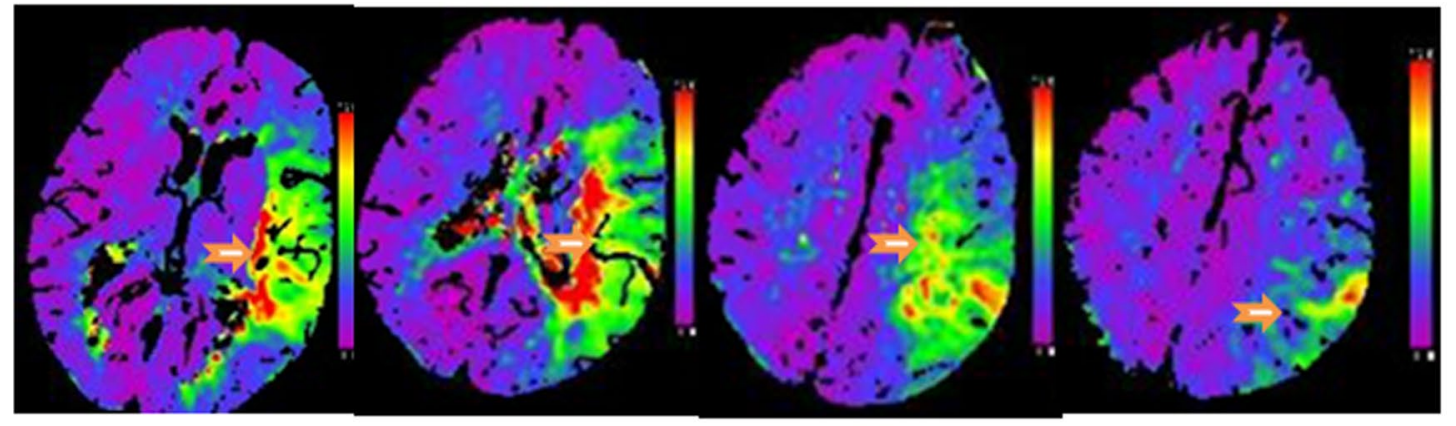

B

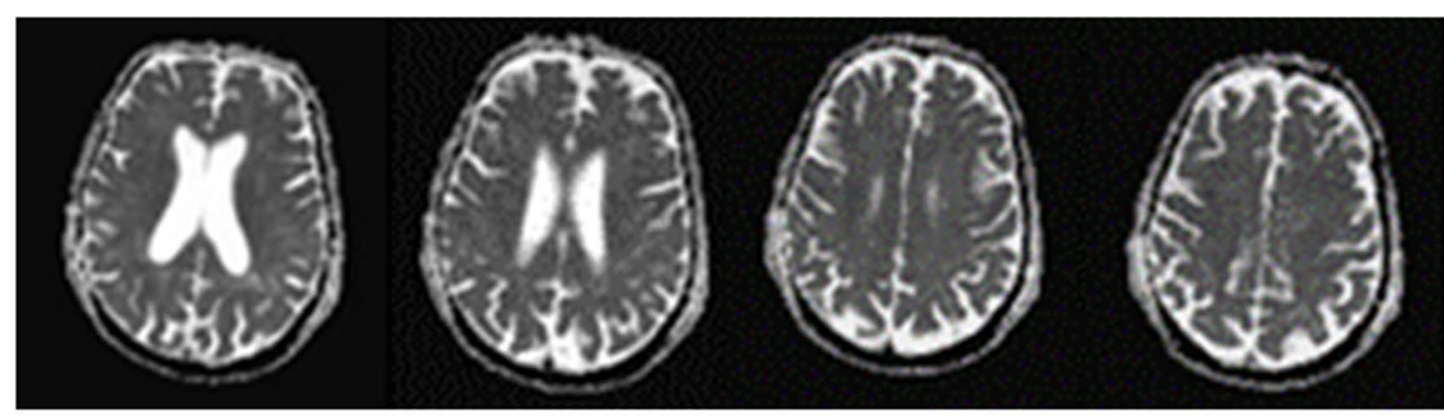

C

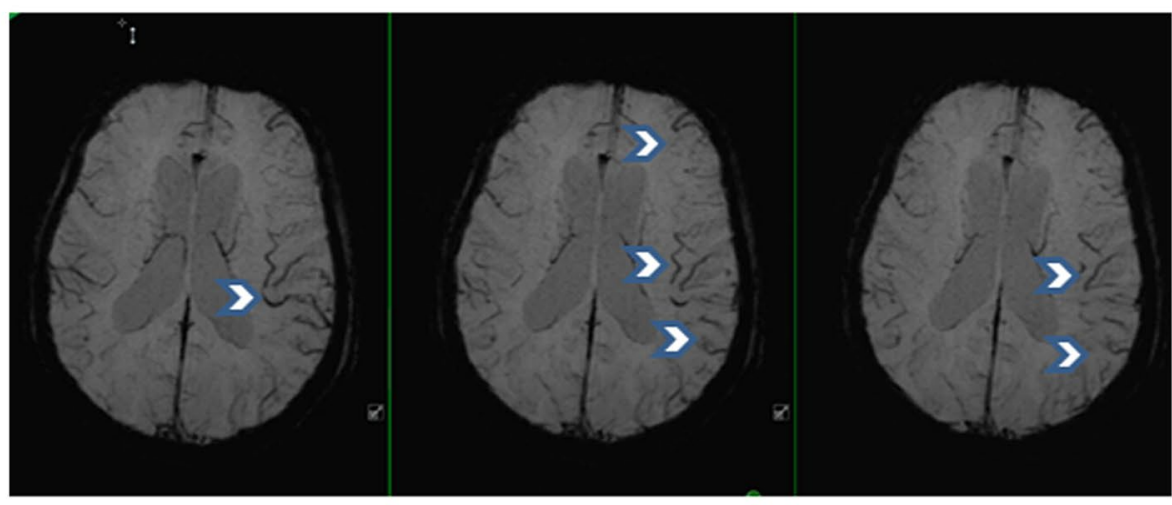

D

Fig. 1 (See legend on previous page.) 


\section{Discussion}

SWI has been increasingly shown to be a useful noncontrast enhanced imaging sequence in the evaluation of acute stroke. One of the most important recent applications of SWI is imaging stroke. It is well known that reliable detection of the ischemic penumbra has a significant impact on treatment and management, especially for acute stroke patients. SWI could be a powerful tool for better detection of the ischemic penumbra [17].

In acute stroke with arterial vessel occlusion, SWI-like sequences may depict clot-related susceptibility changes known as the susceptibility. The SWI plays an important role is detection the area at risk depending on evaluation the asymmetrical prominent vessel sign [18].

In our study, SWI was done for 25 patients and we found that 16 patients had APVS in their images representing $64 \%$ compared to 9 patients who had negative images. These results are in agree with (Chen et al. 2015) who did his study on 22 patients and found that APVS was detected in 15 patient representing about $68 \%$ and 7 patients had negative APVS. They stated that the APVS on SWI is a signature of salvageable ischemic tissue that will become infarcted if blood perfusion cannot be established in time [19].

Luo et al. (2015) reported that the APVS occurred in 11 (61\%) of 18 patients with cerebral infarction on SWI. Their findings showed that a finding of APVS on SWI was significantly associated with the occurrence of damaged vessels or the presence of thrombus in the affected vessels [20].

SWI such as APVS was associated with damaged vessels or the presence of thrombi in the affected vessels. SWI could thus provide information on the status of blood vessels in patients with acute cerebral infarction in addition to that provided by other currently used imaging methods [20].

(Chen et al. 2015) study results showed out of 15 patients with positive APVS 9 of them had arterial occlusion proving that there is great correlation between the APVS and arterial occlusion [19].

In our study, we also found that caudate nucleus was the least affected area; we only found 1 patients had APVS in the caudate nucleus representing about $4 \%$; on the other hand, 15 case showed APVS in M3, 4, 5; thus, they were the most affected areas compared to the deep structures.

These results are in agree with (Chen et al. 2015) who did his study on 22 patients and found that about 9 patients had APVS in the same areas like our study M3, 4, 5 but with lower percentage, and they agree with our study as regarding the least affected area which was caudate nucleus, which can be explained by the admixed venous flow in the thalamostriate vein, which drains not only these structures, but also the thalamus [19].

Jiang et al. 2021 stated that the occurrence rate of APVS was $67.9 \%$ in patients with anterior circulation infarct. Most previous studies on APVS have focused on anterior circulation stroke [21].

All patients with APVS changes had CTP changes so 16 out of 19 patients with CTP had APVS representing 84\%, and only 3 has negative APVS. On the other hand; no one with negative CTP had APVS; there was good correlation between the CTP and APVS with significant $\mathrm{p}$ value.

These results are in agreement with (Wang et al. 2017) and (Wu et al. 2017) who found that there is significance correlation between the CTP and APVS with significant $\mathrm{p}$ value $[22,23]$.

In our study, we used ASPECT to read the CTP, SWI, and DWI to be able to detect the affected areas more easily without bias and we found the mean ASPECT score for SWI $4 \pm 1.4$ and mean aspect for DWI $7.6 \pm 1.2$; in addition, mean aspect for CTP was $4.6 \pm 1.3$. From these results, we found there is significance difference between the SWI and DWI with significant P value. On the other hand, there is no significance difference between the SWI and CTP ASPECT scores.

These results are consistent with research done by Wang et al. (2017) and Wu et al. (2017) who found that there is no significance difference between the SWI and CTP ASPECT scores, but on the other hand there is significance difference between the SWI and DWI ASPECT scores [22, 23].

In our study, only 16 patients with positive APVS showing SWI/ DWI mismatch representing 64\% and 9 patients (36\%) showed negative APVS with no definite mismatch. On the other hand, 19 patients showed prolonged MTT

(See figure on next page.)

Fig. 2 62-year-old male presented to stroke unit with left-sided weakness of 6-h duration. a DWI reveals true diffusion restriction seen involving the M4 \& M5 segments along the territory of MCA with low ADC representing ASPECT score 8 . $\mathbf{b}$ CTP revealed significant delay in mean transient time along the left fronto tempro parietal region (white arrows) along M1 to M6 with involvement of deep nuclei as well as internal capsule compared to right cerebral hemisphere with ASPECT score 3. c SWI of the same patient reveals asymmetrical vessel sign with prominent dark dilated veins on the left cerebral hemisphere along M1, 2, 4, 5 \& M6 segments; however, the deep nuclei are not affected representing ASPECT score 5. The blooming along the affected vessels denotes hemorrhagic transformation (white arrows).The ASPECT score of SWI and perfusion images are lower than that of DWI representing ASPECT score (5, 3 \& 8, respectively). DW/P mismatch noted denoting an area of risk to infarction (penumbra). DW/SWI mismatch is also noted similar to perfusion mismatch 


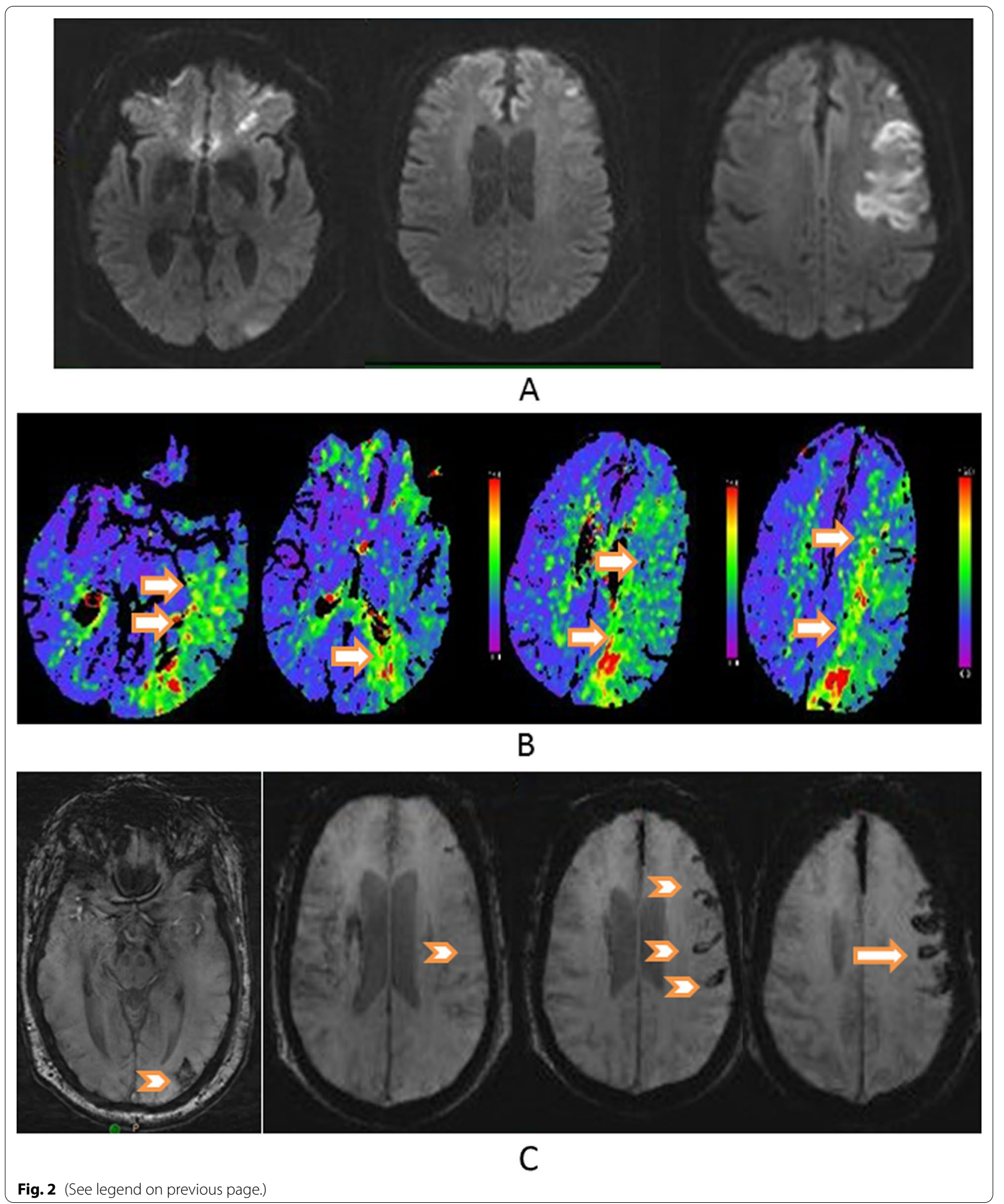




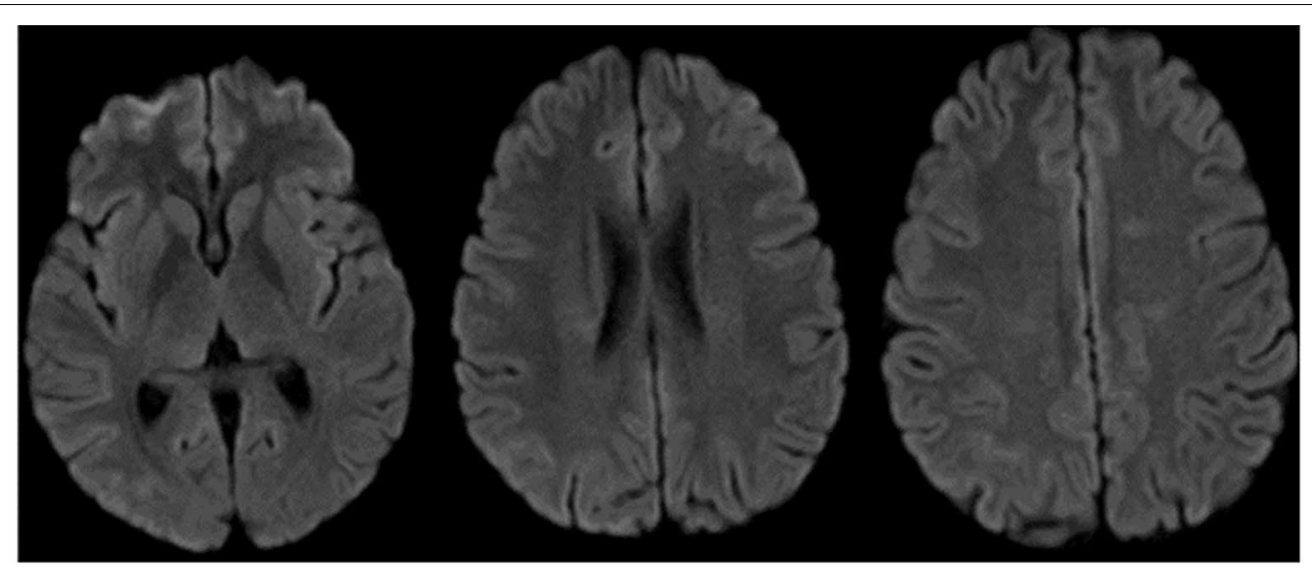

A
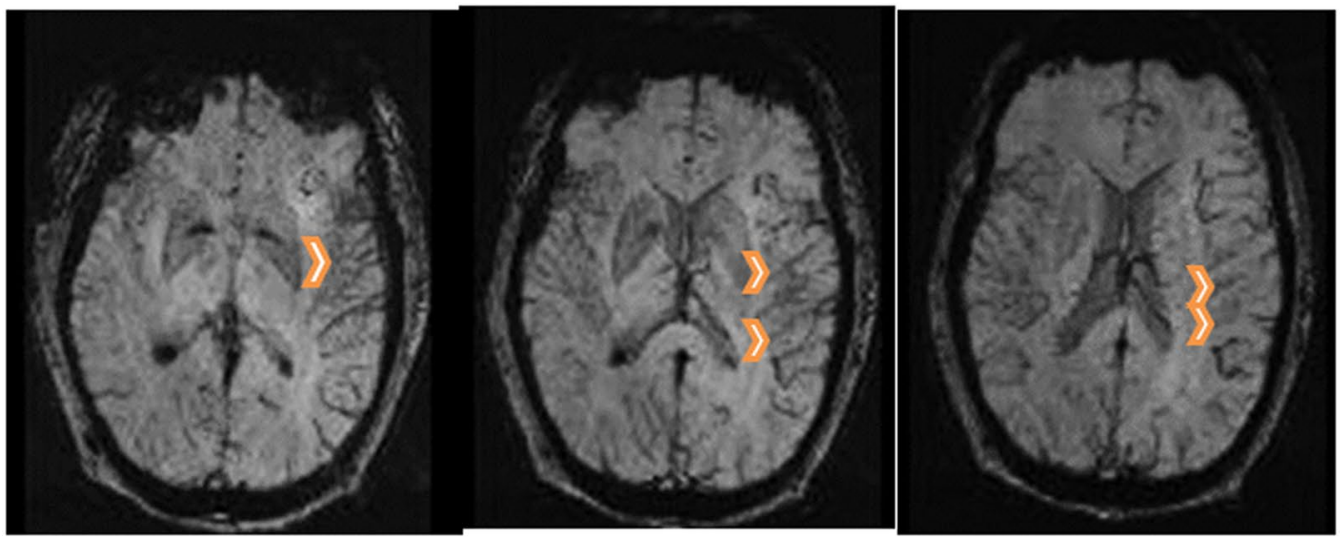

B
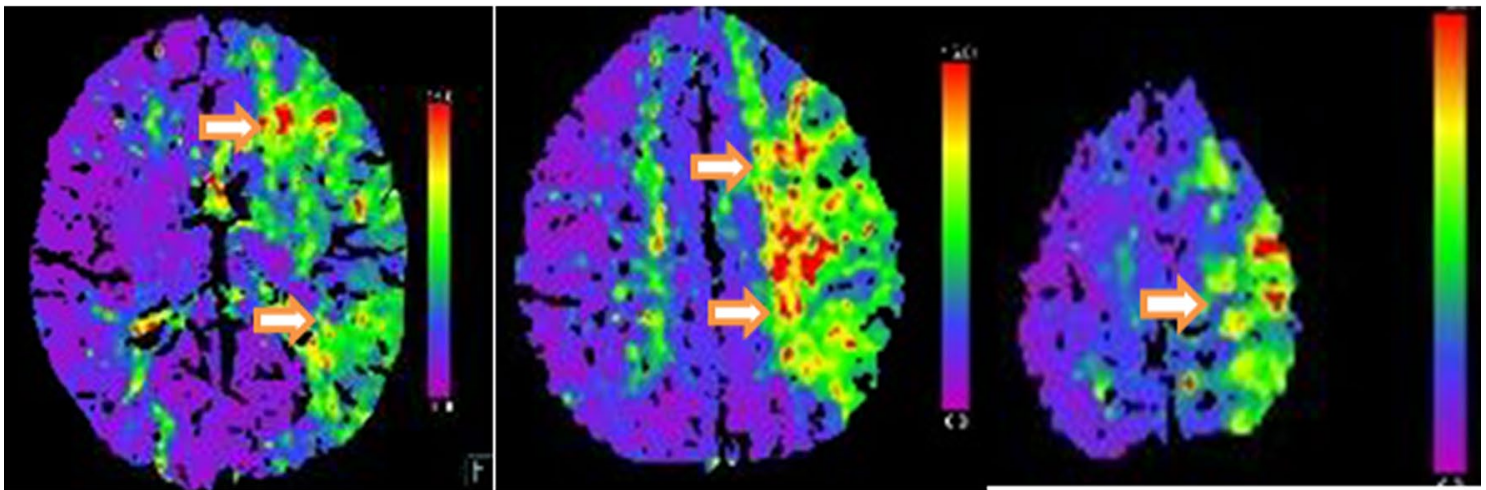

C

Fig. 3 a DWI shows no definite abnormality along the territory of MCA at both sides. b SWI of the same patient revealed asymmetrical vessel sign in the form prominent cortical veins (arrow head) on the left cerebral hemisphere along (M1-M6 segments) representing the area at risk with ASPECT score 4. c CT perfusion of the same patient revealed asymmetry in tissue perfusion between the left and right hemisphere with affected left cerebral hemisphere along (M1-M6 segments) (white arrow) as well as left lentiform nucleus (black arrow) representing the area at risk with aspect score 3 
in CTP representing $76 \%$ and 6 patients have negative results representing $24 \%$. After statistical analysis, there is no significant difference between the SWI/DWI mismatch and CTP/DWI mismatch.

Wang RY 2021 [23] concluded that The MTT is very sensitive to measuring the ischemia state of brain tissue, and it has been used to define the ischemia scope. Several research types have also proved that the increase in oxygen extraction fraction (OEF) is related to the extension of MTT. Since the SWI is sensitive to the change of OEF of deoxy-haemoglobin, SWI can provide a similar metabolic capability to MTT. Furthermore, an SWI-DWI mismatch and a DWI-PWI (MTT) mismatch have similar abilities [24].

Wang et al. 2017 study included 47 patients and they found that only 16 patients had APVS and 19 patients had positive perfusion finding with SWI/DWI mismatch that is correlated with CTP/DWI mismatch with no significant difference between the SWI and CTP in detection of area at risk which is in agreement with our research [22]

A study done by Liu et al. 2016 showed that SWI/DWI is positively correlated with PWI/DWI and PWI-DWI mismatch volumes; their results indicated that SWI-DWI mismatch has the ability to detect penumbra [25].

Wang RY 2021 who did their research on 52 patients found that 32 patients have DWI-SWI mismatches; they stated that by contrasting the DWI-SWI mismatch with the DWI-PWI mismatch in evaluating the ischemic penumbra and found that a DWI-SWI mismatch has a similar ability as a DWI-PWI to detect the ischemic penumbra, which indicates that SWI can be used to forecast the ischemic penumbra of patients with acute ischemic cerebral stroke; they concluded also that increased and thickened venous shadows in the SWI sequence of the hypoperfusion area compared with the contralateral [24].

In addition, Luo et al. 2015 found that DWI-SWI mismatch was positively correlated with the DWI-MTT mismatches. These findings suggested that similar to the DWI-MTT mismatch, the DWI-SWI mismatch could reflect the size of ischemic penumbra in patients with cerebral infarction [20].

However, Verma et al. found that although SWI is helpful in detecting tissue at risk, it cannot replace PWI. The reason might be that MTT detects more illperfused areas than SWI, especially in good collateralized subjects [26, 27].

SWI is a technique that is contrast independent, consuming less time, and can be considered as an alternative to CT Perfusion valuable in detecting penumbra and help to rapidly directing patients indicated for thrombolytic therapy.

\section{Conclusion}

SWI is a promising technique and comparable to CT perfusion is evaluation of penumbra in the settings of acute infarction.

\section{Acknowledgements \\ We gratefully acknowledge the work efficiency and devotion of our imaging technicians which made this work possible.}

\section{Authors' contributions}

All authors have read and approved the current study. MA took part in study design, manuscript writing, image interpretation. MFA participated in manuscript revision, image interpretation, statistical analysis. AM involved in manuscript revision, image interpretation. AK took part in image revision, manuscript editing, and image interpretation. MFG participated in manuscript revision and editing, patient referee. MA, MFA, AMY, AK, MFG: All authors read and approved the manuscript.

\section{Funding}

No sources of funding.

\section{Availability of data and materials}

The datasets used and or analysed during the current study are available for the corresponding author on reasonable request.

\section{Declarations}

\section{Ethics approval and consent to participate}

This study was approved by the research ethics committee of Faculty of medicine, at Minia University in Egypt on 2020 (reference number not applicable). All patients in this study gave a written consent to participate in this research project.

\section{Consent for publication}

Informed consent is given to publish data contained in this study.

\section{Competing interests}

The authors declare that they have no competing interests.

\section{Author details}

${ }^{1}$ Radiology Department, Faculty of Medicine, Minia University, Minya, Egypt.

${ }^{2}$ Radiology Department, Faculty of Medicine, South Valley University, Qina,

Egypt.

Received: 29 June 2021 Accepted: 26 September 2021

Published online: 06 October 2021

\section{References}

1. Feigin VL, Forouzanfar MH, Krishnamurthi R, Mensah GA, Connor M, Bennett DA et al (2014) Global and regional burden of stroke during 1990-2010: findings from the Global Burden of Disease Study 2010. Lancet 383:245-254

2. Fisher M, Bastan B (2012) Identifying and utilizing the ischemic penumbra. Neurology 79:S79-85

3. Jung S, Gilgen M, Slotboom J, El-Koussy M, Zubler C, Kiefer C, Luedi R et al (2013) Factors that determine penumbral tissue loss in acute ischaemic stroke. Brain 136:3554-3560

4. Olivot JM, Mlynash M, Thijs VN, Kemp S, Lansberg MG, Wechsler L et al (2009) Optimal Tmax threshold for predicting penumbral tissue in acute stroke. Stroke 40:469-475

5. Kakuda W, Lansberg MG, Thijs VN, Kemp SM, Bammer R, Wechsler LR et al (2008) Optimal definition for PWI/DWI mismatch in acute ischemic stroke patients. J Cereb Blood Flow Metab 28:887-891

6. Astrup J, Siesjö BK, Symon L (1981) Thresholds in cerebral ischemia-the ischemic penumbra. Stroke 12(6):723-725 
7. Kao H-W, Tsai FY, Hasso AN (2012) Predicting stroke evolution: comparison of susceptibility-weighted MR imaging with MR perfusion. Eur Radiol 22(7):1397-1403

8. von Kummer R, Bourquain H, Bastianello S, Bozzao L (2001) Early prediction of irreversible brain damage after ischemic stroke at CT. Radiology 219:95-100

9. Sarikaya B, Provenzale J (2010) Frequency of various brain parenchymal findings of early cerebral ischemia on unenhanced CT scans. Emerg Radiol 17:381-390

10. Mittal S, Wu Z, Neelavalli J, Haacke EM (2009) Susceptibility-weighted imaging: technical aspects and clinical applications, part 2. Am J Neuroradiol 30(2):232-252

11. Barber P, Darby D, Desmond P, Yang Q, Gerraty R, Jolley D et al (1998) Prediction of stroke outcome with echoplanar perfusion-and diffusionweighted MRI. Neurology 51(2):418-426

12. Parsons MW, Yang Q, Barber PA, Darby DG, Desmond PM, Gerraty RP et al (2001) Perfusion magnetic resonance imaging maps in hyperacute stroke: relative cerebral blood flow most accurately identifies tissue destined to infarct. Stroke 32(7):1581-1587

13. Haacke EM, Xu Y, Cheng YC, Reichenbach JR (2004) Susceptibility weighted imaging (SWI). Magn Reson Med 52(3):612-618

14. Sehgal V, Delproposto Z, Haacke EM, Tong KA, Wycliffe N, Kido DK et al (2005) Clinical applications of neuroimaging with susceptibility-weighted imaging. J Magn Resonan Imaging 22(4):439-450

15. Tsui Y-K, Tsai FY, Hasso AN, Greensite F, Nguyen BV (2009) Susceptibilityweighted imaging for differential diagnosis of cerebral vascular pathology: a pictorial review. J Neurol Sci 287(1-2):7-16

16. Grubb RL Jr, Derdeyn CP, Fritsch SM, Carpenter DA, Yundt KD, Videen TO et al (1998) Importance of hemodynamic factors in the prognosis of symptomatic carotid occlusion. JAMA 280(12):1055-1060

17. Haacke EM, Mittal S, Wu Z, Neelavalli J, Cheng YC (2009) Susceptibilityweighted imaging: technical aspects and clinical applications, part 1. AJNR Am J Neuroradiol 30(1):19-30

18. Quintana F (2008) CT protocol for acute stroke: tips and tricks for general radiologists1. Radiographics 28:1673-1687

19. Meoded A, Poretti A, Benson JE et al (2014) Evaluation of the ischemic penumbra focusing on the venous drainage: the role of susceptibility weighted imaging (SWI) in pediatric ischemic cerebral stroke. J Neuroradiol 41(2):108-116
20. Sven H, Mark H, Majda MT, Frederik B (2021) Susceptibility-weighted imaging: technical essentials and clinical neurologic applications. Radiology 299:3-26

21. Chen C-Y, Chen C-I, Tsai FY, Tsai P-H, Chan WP (2015) Prominent vessel sign on susceptibility-weighted imaging in acute stroke: prediction of infarct growth and clinical outcome. PLoS ONE 10(6):e0131118

22. Luo S, Yang L, Wang L (2015) Comparison of susceptibility-weighted and perfusion-weighted magnetic resonance imaging in the detection of penumbra in acute ischemic stroke. J Neuroradiol 42:255-260

23. Jiang HF, Zhang Y, Pang JX, Shao P, Qiu HC, Liu A et al (2021) Factors associated with prominent vessel sign on susceptibility-weighted imaging in acute ischemic stroke. Sci Rep 11(1):5641

24. Wang T, Zhu L, Hu C, Gong S, Jiang H, Chen H et al (2017) The diagnostic value of susceptibility-weighted imaging for ischemic penumbra in patients with acute ischemic stroke. Technol Health Care 25(S1):449-457

25. Wu X, Luo S, Wang Y, Chen Y, Liu J, Bai J, et al. Use of susceptibilityweighted imaging in assessing ischemic penumbra: a case report. Medicine. 2017;96(6)

26. Wang RY, Li ZW, Huang W, Yang HQ, Gao B, Chen TY (2021) The value of susceptibility-weighted imaging (SWI) in evaluating the ischemic penumbra of patients with acute cerebral ischemic stroke. Neuropsych Disease Treat 17:1745-1750

27. Liu H, Mei W, Li Y, Huang Y, Ruan S, Zhang Q (2016) Zhang J Susceptibility-diffusion mismatch: an effective method by which to detect perfusion-diffusion mismatch in acute ischemic stroke. Int J Clin Exp Med 9(10):19691-19699

28. Verma RK, Hsieh K, Gratz PP et al (2014) Leptomeningeal collateralization in acute ischemic stroke: impact on prominent cortical veins in susceptibility-weighted imaging. Eur J Radiol 83:1448-1454

29. Baik SK, Choi W, Oh SJ et al (2012) Change in cortical vessel signs on susceptibility-weighted images after full recanalization in hyperacute ischemic stroke. Cerebrovasc Dis 34:206-212

\section{Publisher's Note}

Springer Nature remains neutral with regard to jurisdictional claims in published maps and institutional affiliations.

\section{Submit your manuscript to a SpringerOpen ${ }^{\circ}$ journal and benefit from:}

- Convenient online submission

- Rigorous peer review

- Open access: articles freely available online

- High visibility within the field

- Retaining the copyright to your article

Submit your next manuscript at $\boldsymbol{\nabla}$ springeropen.com 